\section{PTU-110 OUTCOMES OF INADEQUATE BOWEL PREPARATION COLONOSCOPY: SINGLE-CENTRE RETROSPECTIVE ANALYSIS}

1,2Keith Siau*, 'Kirsty Carrothers, ${ }^{1}$ Sharan Shetty. ${ }^{1}$ Endoscopy Unit, Dudley Group Hospitals NHSFT, Dudley; ${ }^{2}$ University of Birmingham, Birmingham

\subsection{6/gutjnl-2019-BSGAbstracts.99}

Introduction Quality in colonoscopy is underpinned by the quality of bowel preparation, however, data on real-world management following inadequate bowel preparation remain sparse. We aimed to audit the rates and outcomes of inadequate bowel preparation on colonoscopy, including subsequent investigations and rates of post-colonoscopy colorectal cancer (PCCRC).

Methods In this single-centre audit, all colonoscopies performed in 2015 were identified from an endoscopy reporting database. Patients with colonic resection were excluded. Bowel preparation was measured using the Aronchick scale and considered inadequate if reported as poor $(<90 \%$ mucosal visualisation) despite washing. Retrospective follow-up was performed through electronic healthcare records with primary care linkage to identify subsequent investigations and rates of 3 yr-PCCRC. Subsequent investigation was defined as a repeat colonoscopy or relevant imaging within 6 months of an index procedure with inadequate preparation. Multivariable binary logistic regression was undertaken to identify predictors of subsequent investigation. Comparisons were performed at endoscopist-level and between adequate and inadequate bowel preparation groups using chi-square and Mann-Whitney tests.

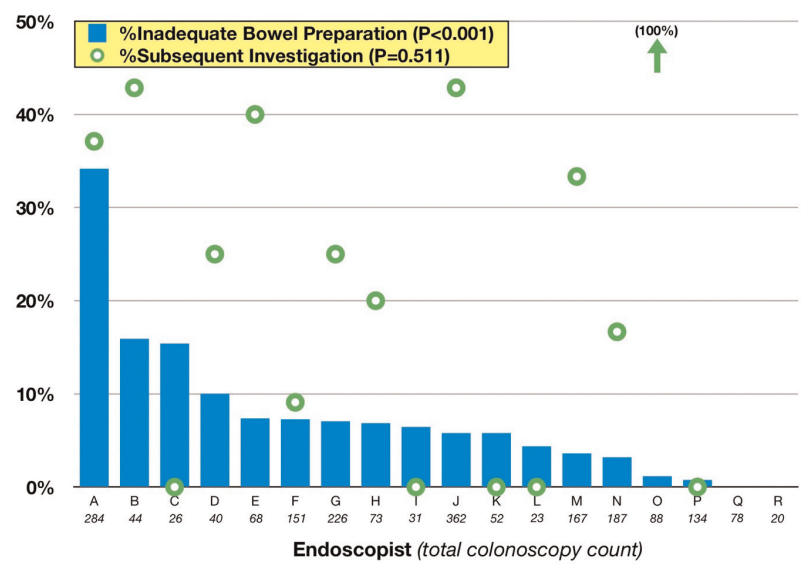

Abstract PTU-110 Figure 1

Results Of 2305 procedures recorded over the study period, inadequate preparation was indicated in 235 (10.2\%). This cohort was associated with lower caecal intubation rates $(80.7 \%$ vs. $92.3 \%, \mathrm{P}<0.001)$, higher polyp detection rates $(47.7 \%$ vs $40.9 \%, \mathrm{P}=0.04)$ but no differences in median age (65 vs. $64, \mathrm{P}=0.190)$ or polyp resection rates $(37.0 \%$ vs. $34.8 \%, \mathrm{P}=0.484)$. Subsequent investigations were performed in 63 patients $(26.8 \%)$, comprising repeat colonoscopy $(\mathrm{N}=31)$, CT imaging $(\mathrm{N}=31)$ and barium enema $(\mathrm{N}=1)$. Of the 18 endoscopists identified with $>20$ procedures in 2015, the rates of inadequate preparation ranged between endoscopists $(0 \%-34.2 \% ; \mathrm{P}<0.001)$, although there was no significant difference in the practice of subsequent investigation (range $0-$ 100\%, $\mathrm{P}=0.511$ ) [Abstract PTU110 figure 1]. On multivariable analysis, factors independently associated with subsequent investigations included: non-completion colonoscopy (OR 7.6, $\mathrm{P}<0.001$ ), any abnormal diagnosis (OR 2.4, $\mathrm{P}=0.034)$, and younger age $(\mathrm{P}=0.040)$. No cases of $3 \mathrm{yr}-$ PCCRC were identified within the cohort.

Conclusion Although the outcome of inadequate bowel preparation is an established quality metric in colonoscopy, its interpretation appears to vary between endoscopists, with the practice of instigating subsequent investigations also varying at procedural and patient level. Whilst our data should prompt reflection by local and national quality assurance groups, more robust studies are required to determine the impact of inadequate bowel preparation on patient outcomes such as PCCRC.

\section{PTU-111 DOES A 2 WEEK BREAK FROM COLONOSCOPY PRACTICE IMPACT ON PERFORMANCE?}

\begin{abstract}
${ }^{1,2}{ }^{2}$ Keith Siau ${ }^{*},{ }^{3}$ Ben Disney, ${ }^{4}$ Danny Cheung, ${ }^{5}$ Aravinth Murugananthan, ${ }^{6}$ Deevia Kotecha, ${ }^{6}$ Ajay Verma, ${ }^{7}$ Mo Thoufeeq, ${ }^{1}$ Sharan Shetty. ${ }^{1}$ Dudley Group Hospitals NHSFT, Dudley, UKi ${ }^{2}$ University of Birmingham, Birmingham; ${ }^{3}$ University Hospital Coventry, Coventry; ${ }^{4}$ Worcester Acute Hospitals NHS Trust, Worcester; ${ }^{5}$ New Cross Hospital, Wolverhampton; ${ }^{6}$ Kettering General Hospitals NHSFT, Kettering; ${ }^{7}$ Sheffield Teaching Hospitals NHSFT, Sheffield
\end{abstract}

\subsection{6/gutjnl-2019-BSGAbstracts. 100}

Introduction Although breaks in colonoscopy practice have been shown to adversely affect trainee performance, ${ }^{1}$ this has not been studied in independent endoscopists. We aimed to evaluate the impact of $\mathrm{a} \geq 2 \mathrm{wk}$ break in colonoscopy on the completion metrics of independent practitioners.

Methods We performed a retrospective analysis of electronic colonoscopy databases in six NHS Trusts within East Midlands, West Midlands and South Yorkshire. Procedures between 2016-2018 were extracted by endoscopist identifier and in time order. A break was defined as a $14 \mathrm{~d}+$ interval between procedures. For each break, 20 pre and 20 post break procedures were identified. Breaks were excluded if a $\geq 2$ wk interval occurred within the 20 pre-break procedures, and if any of the 20 pre-break procedures occurred within $14 \mathrm{~d}$ of another break or overlapped with any post-break procedures. Study outcomes included: 1) the Performance Indicator of Colonic Intubation (PICI), ${ }^{2}$ a composite endpoint integrating completion, sedation and discomfort metrics, and 2) the unadjusted caecal intubation rate (CIR). Pairwise comparisons between pre and post break data were made for pooled data and by endoscopist using Wilcoxon signed rank tests.

Results 352 breaks undertaken by 113 endoscopists were eligible for analysis, comprising 14,080 procedures. The median break interval was 18d (IQR 14-24). Baseline comparisons of the pre and post groups revealed no significant differences in age, gender or proportion of Bowel Cancer Screening cases. Between pre and post break periods, mean PICI fell from $82.1 \%$ to $80.0 \%(\mathrm{P}<0.001)$ and mean CIR from $93.8 \%$ to 92.5\% $(\mathrm{P}=0.003)$. Trends in PICI with 10 -procedure moving averages relative to the break (Abstract PTU111 figure 1) did not suggest reversion to baseline rates after 20 postbreak procedures. At endoscopist-level, breaks were associated with a small but significant reductions in median PICI $(86.7 \%$ to $85.0 \%, \mathrm{P}<0.001)$ and $\mathrm{CIR}(95.0 \%$ to $92.5 \%, \mathrm{P}=0.004)$, with $58.4 \%$ of endoscopists demonstrating a decrement in PICI. There was no significant correlation between the 


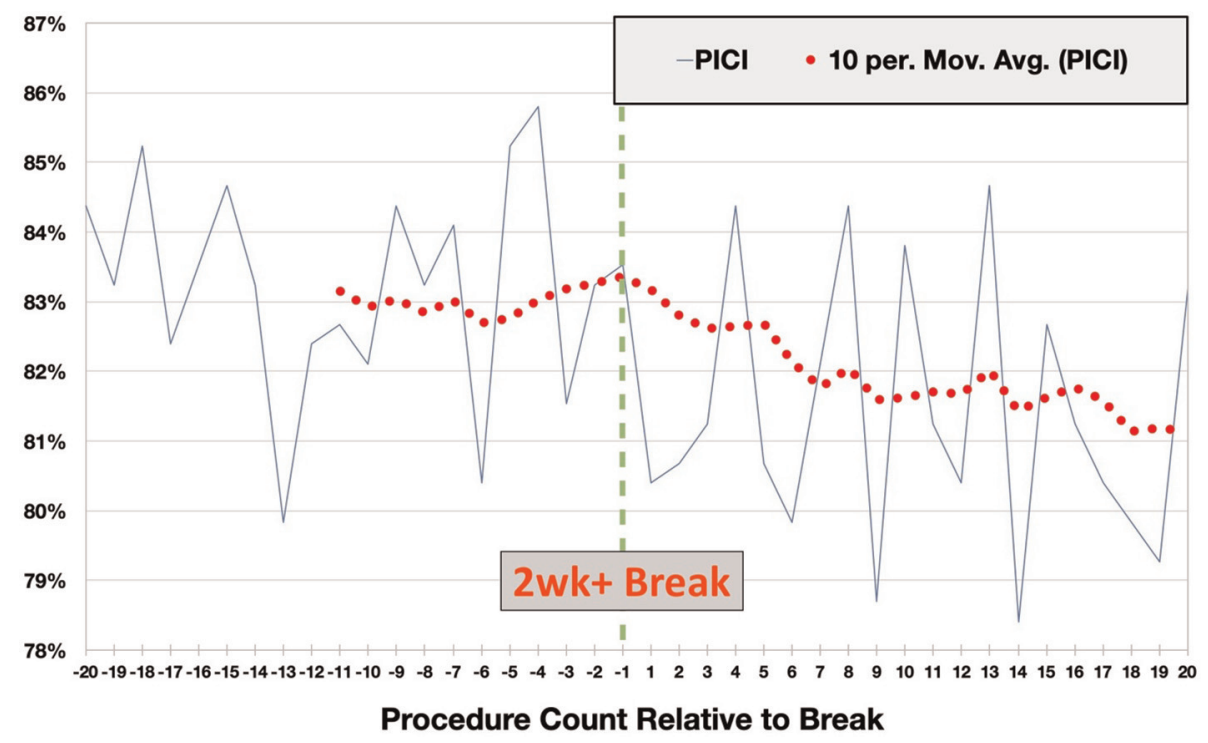

Abstract PTU-111 Figure 1

duration of the break and the difference in PICI (Pearson coefficient $-0.014, \mathrm{P}=0.788$ ).

Conclusions A break in practice of $\geq 2 \mathrm{wks}$ appears to result in a small but statistically significant decrement in colonoscopy completion metrics as measured by PICI and CIR.

\section{REFERENCES}

1. Jorgensen JE. Gastrointest Endosc 2013; 78:503-9.

2. Valori RM. Endoscopy 2018;50:40-51.

\section{PTU-112 RISK FACTORS FOR EROSIVE TOOTH WEAR IN PATIENTS WITH GORD:A PROSPECTIVE CROSS-SECTIONAL CASE CONTROL STUDY}

1,2Rasha alharthi*, 'David bartlett, ' Jafar jafari, 'Rebecca moazzez. ' King's College London, London, UK; ${ }^{2}$ Princess Noura Bnt Abdulrahman University, Riyadh, Saudi Arabia

\subsection{6/gutjnl-2019-BSGAbstracts.101}

Introduction Some patients with GORD develop ETW referred to as GE(GORD-Erosion group) whereas others don't and these are referred to as GNE(GORD-no-erosion group).The latest gold standard reflux monitoring is impedance-pH monitoring to identify the type of reflux and correlate the symptoms to each type of reflux. Basic Erosive Tooth Wear Examination (BEWE) is a scoring system to classify the affected tooth surfaces. This study used these two techniques which have not been used previously aiming to assess the risk factors and predictive values associated with presence of ETW in patients with GORD.

Methods Patients referred for assessment of GORD to the Oesophageal Laboratory at Guy's Hospital were recruited (REC Ref 18/NE/0099). Participants were consented and given a validated Reflux Symptom Questionnaire 7-day recall (RESQ) to assess the frequency and intensity of GORD symptoms. Data reported from the impedance results were: total acid exposure, acid exposure in upright position and acid exposure in supine position. A clinical assessment of ETW was done using BEWE; a validated index dividing the mouth into 6 areas and each is scored from 0 to $3(0=$ no ETW, $1=$ initial loss of surface texture, $2=$ loss of hard tissue $<50 \%$ of surface area and $3=$ loss of hard tissue $\geq 50 \%$ of surface area). Those with a cumulative score of $\geq 12$ and at least 1 oral area scoring 3 were included in the GE-group opposed to those scoring $<12$ were included in the GNE-group.

Data were not normally distributed and hence median (IQR), Kruskal-Wallis and ROC curve were applied, $(\mathrm{P}<0.05)$ considered significant.

Results RESQ-7: 151 GORD patients recruited: GE( $\mathrm{n}=76)$, $\mathrm{GNE}(\mathrm{n}=75)$. Median(IQR) frequency and intensity of GORD for hoarseness, cough and difficulty swallowing were significantly different between groups: frequency[GNE:7(2,11.5) and $\mathrm{GE}: 12.5(2,17.5) ; \mathrm{P}=0.007], \quad$ intensity[GNE:4(2,7)and $\mathrm{GE}: 6.6$ $(3,10) ; \mathrm{P}=0.006]$. ROC curve for the total frequency and intensity illustrates that patients with total frequency an intensity of $\geq 26$ and $\geq 37$ most probably will have ETW, respectively.

Impedence-pH-monitoring patients reported: heartburn, chestpain, regurgitation, cough, hoarseness, acid taste in the mouth, throat acid, belching and epigastric pain, with no statistical significance between the GE and GNE groups. Total acid exposure and acid exposure in upright position were highly significant when comparing $\mathrm{GE}(\mathrm{n}=53)$ and $\mathrm{GNE}(\mathrm{n}=56)$ $(\mathrm{P}=0.0007)$.

Conclusion The risk of developing ETW is frequency score of 26 and above using the RESQ-7 and intensity score of 37 and above. Hoarseness, coughing and difficulty in swallowing were the significant risk factors for presence of ETW in patients with GORD. These atypical symptoms of GORD may indicate the presence of acid higher up in the oesophagus and therefore more likelihood of acid reaching the mouth.

\section{PTU-113 HOW COMMONLY IS PANCREATIC CANCER DIAGNOSED FOLLOWING AN ENDOSCOPIC ULTRASOUND THAT DID NOT DIAGNOSE CANCER?}

${ }^{1}$ Dominic King*, ${ }^{2}$ Amandeep Dosanjh, ${ }^{2}$ Prashant Patel, ${ }^{3}$ John Leeds, ${ }^{3}$ Manu Nayar ${ }^{3}$ Kofi Oppong, ${ }^{1}$ Nigel Trudgill. ${ }^{1}$ Sandwell and West Birmingham NHS Trust, Birmingham, UK; ${ }^{2}$ University Hospital Birmingham, Birmingham, UK; ${ }^{3}$ Newcastle upon Tyne Hospitals NHS Foundation Trust, Newcastle, UK

10.1136/gutjnl-2019-BSGAbstracts. 102 